\title{
Multiple return times theorems for weakly mixing systems
}

by

\author{
I. ASSANI \\ Department of Mathematics, University of North Carolina at Chapel Hill, \\ Chapel Hill, NC 27599, USA
}

Received in 15 June 1998, revised 16 June 1999

ABSTRACT. - We prove the pointwise convergence of weighted averages $\frac{1}{N} \sum_{n=1}^{N} a_{n} g\left(R^{n} z\right)$ where $(Z, \mathcal{K}, \nu, R)$ is an ergodic dynamical system. The sequence $a_{n}$ is given by expression of the form

$$
a_{n}=a_{n}\left(x, y_{1}, y_{2}, \ldots, y_{J}\right)=\left(\prod_{i=1}^{H} f_{i}\left(T^{b_{i} n} x\right)\right) \cdot\left(\prod_{j=1}^{J} g_{j}\left(S_{j}^{n} y_{j}\right)\right),
$$

where $\left(b_{1}, b_{2}, \ldots, b_{H}\right) \in \mathbb{Z}^{H}$ and $J$ is a positive integer.

The functions $f_{i}$ and $g_{j}$ are bounded and the systems $(X, \mathcal{F}, \mu, T)$ and $\left(Y_{j}, \mathcal{G}_{j}, m_{j}, S_{j}\right)$ are weakly mixing. $\odot 2000$ Éditions scientifiques et médicales Elsevier SAS

RÉSUMÉ. - Nous montrons la convergence ponctuelle des moyennnes pondérées

$$
\frac{1}{N} \sum_{n=1}^{N} a_{n} g\left(R^{n} z\right)
$$

où $(Z, \mathcal{K}, v, R)$ est un système ergodique dynamique. La suite $a_{n}$ est obtenue par des expressions de la forme

$$
a_{n}=a_{n}\left(x, y_{1}, y_{2}, \ldots, y_{J}\right)=\left(\prod_{i=1}^{H} f_{i}\left(T^{b_{i} n} x\right)\right) \cdot\left(\prod_{j=1}^{J} g_{j}\left(S_{j}^{n} y_{j}\right)\right),
$$


où $\left(b_{1}, b_{2}, \ldots, b_{H}\right) \in \mathbb{Z}^{H}$ et $J$ est un entier positif.

Les fonctions $f_{i}$ et $g_{j}$ sont bornées, les systèmes $(X, \mathcal{F}, \mu, T)$ et $\left(Y_{j}, \mathcal{G}_{j}, m_{j}, S_{j}\right)$ sont faiblement mélangeants. ๑ 2000 Éditions scientifiques et médicales Elsevier SAS

\section{INTRODUCTION}

Let $(Z, \mathcal{K}, v, R)$ be an ergodic dynamical system on a probability measure space $(Z, \mathcal{K}, v)$ and $\left(a_{n}\right)$ a sequence of scalars.

DEFINITION. - The sequence $\left(a_{n}\right)$ is a good universal weight for the pointwise convergence if for all ergodic dynamical systems $(Z, \mathcal{K}, v, R)$ and all $g \in L^{\infty}$ the averages

(0) $\frac{1}{N} \sum_{n=1}^{N} a_{n} g\left(R^{n} z\right)$ converge a.e. (v) when $N$ tends toward infinity.

This definition was used in [7] where a nice survey of weighted averages is given (up to 1985).

We are interested in weights selected randomly. In the case $a_{n}=$ $\mathbf{1}_{A}\left(T^{n} x\right), n \geqslant 1$, where $(X, \mathcal{F}, \mu, T)$ is an ergodic dynamical system and $A$ a measurable set, it was proved in [4] and [6] that this sequence provides (a.e. $\mu$ ) a universal good weight for the pointwise convergence. This sequence $\left(\mathbf{1}_{A}\left(T^{n} x\right)\right)$ is a sequence of 0 and 1 . The ergodicity of $T$ guarantees that for a.e. $x$ this sequence of 0 and 1 has positive density. We can write these averages as

$$
\frac{1}{N} \sum_{n=1}^{N} \mathbf{1}_{A}\left(T^{n} x\right) g\left(R^{n} z\right)=\frac{1}{N} \sum_{k_{n}(x) \leqslant N} g\left(R^{k_{n}(x)} z\right),
$$

where $k_{n}(x)$ is the $n$th return time of $T^{\ell} x$ to the set $A$.

We showed in [2] that much more is true on this kind of return times property, where a single null set "works" for an uncountable number of dynamical systems. At the same time the study in [2], motivated by the range of the functions involved, lead to the introduction of a new maximal function namely,

$$
N^{*} f(x)=\sup _{n} \frac{\#\left\{k: \frac{f\left(T^{k} x\right)}{k} \geqslant \frac{1}{n}\right\}}{n} .
$$


The use of this maximal function lead also to the first multidimensional return time result for $L^{1}$ Bernouilli processes.

In this paper we are interested in extending this [6] result simultaneously in two directions. This extension is not just for the sake of generalization. One of the motivations behind this is primarily the use and understanding of the structure of pairwise independent joinings and also to study the possibility of averaging along nonstationary sampling. Let us consider the simplest case of the averages

$$
\frac{1}{N} \sum_{n=1}^{N} \mathbf{1}_{A}\left(T^{n} x\right) \mathbf{1}_{B}\left(T^{2 n} x\right) g\left(R^{n} z\right),
$$

where $A$ and $B$ are measurable sets and $(X, \mathcal{F}, \mu, T)$ is weakly mixing. (The case $T$ ergodic for the averages in (2) is studied in [1].) By the "double a.e. recurrence" result obtained in [5] we know that the averages $\frac{1}{N} \sum_{n=1}^{N} \mathbf{1}_{A}\left(T^{n} x\right) \mathbf{1}_{B}\left(T^{2 n} x\right)$ converge a.e. to $\mu(A) \mu(B)$. This means that the sequence $\left(\mathbf{1}_{A}\left(T^{n} x\right) \cdot \mathbf{1}_{B}\left(T^{2 n} x\right)\right)$ also has a positive density. We can remark that the functions $f_{n}: f_{n}(x)=\mathbf{1}_{A}\left(T^{n} x\right) \cdot \mathbf{1}_{B}\left(T^{2 n} x\right)$ do not necessarily have the same distribution. Hence contrary to (1) the sequence $a_{n}(x)=\mathbf{1}_{A}\left(T^{n} x\right) \mathbf{1}_{B}\left(T^{2 n} x\right)$ is not given by a sampling of a stationary process. In [3] we proved that for "many" weakly mixing systems $(X, \mathcal{F}, \mu, T)$ for all positive integers $H$, the averages $\frac{1}{N} \sum_{n=1}^{N} \mathbf{1}_{A_{1}}\left(T^{n} x\right) \mathbf{1}_{A_{2}}\left(T^{2 n} x\right) \cdots \mathbf{1}_{A_{H}}\left(T^{H n} x\right)$ converge a.e. to $\prod_{i=1}^{H} \mu\left(A_{i}\right)$ (where $A_{i}, 1 \leqslant i \leqslant H$, are measurable subsets of $X$ ). Such is the case for $\mathrm{K}$ automorphisms (see also [10]) and Chacon famous rank one map. We will extend this result by showing that for such weakly mixing systems the sequence $a_{n}(x)=\prod_{i=1}^{H} \mathbf{1}_{A_{i}}\left(T^{b_{i} n} x\right)$ is $\mu$ a.e., a good universal weight. The proof given in [3] shows that the averages $\frac{1}{N} \sum_{n=1}^{N} \prod_{i=1}^{H} f_{i}\left(T^{b_{i} n} x\right)$ converge a.e. for all $\left(b_{1}, b_{2}, \ldots, b_{H}\right) \in \mathbb{Z}^{H}$ and if the $b_{i}$ are all distinct and not equal to zero then the limit is equal to $\prod \int f_{i} \mathrm{~d} \mu$. To simplify the notation we introduce the following definition.

DEFINITION. - Let $(X, \mathcal{F}, \mu, T)$ be a weakly mixing dynamical system. We say that this system is a.e. multiple recurrent if for all $H$ positive integer, for all $\left(f_{1}, f_{2}, \ldots, f_{H}\right) \in L^{\infty}(\mu)$, for all $\left(b_{1}, b_{2}, \ldots, b_{H}\right) \in \mathbb{Z}^{H}$, $b_{i}$ distinct and not equal to zero, the sequence

$$
\frac{1}{N} \sum_{n=1}^{N}\left(\prod_{i=1}^{H} f_{i}\left(T^{b_{i} n} x\right)\right) \text { converges a.e. to } \prod_{i=1}^{H} \int f_{i} \mathrm{~d} \mu .
$$


As indicated in [3] the problem of the a.e. convergence of the averages

$$
\frac{1}{N} \sum_{n=1}^{N}\left(\prod_{i=1}^{H} f_{i}\left(T^{b_{i} n} x\right)\right)
$$

was introduced by $\mathrm{H}$. Furstenberg in [8].

Thus by considering weights of the form $a_{n}(x)=\prod_{i=1}^{H} \mathbf{1}_{A_{i}}\left(T^{b_{i} n} x\right)$ we will get an extension in one direction. This extension already generalizes our result in [3]. In the second direction we will look at weights of the form

$$
a_{n}\left(x, y_{1}, y_{2}, \ldots, y_{J}\right)=\left(\prod_{i=1}^{H} \mathbf{1}_{A_{i}}\left(T^{b_{i} n} x\right)\right) \cdot\left(\prod_{j=1}^{J} g_{j}\left(S_{j}^{n} y_{j}\right)\right),
$$

where $(X, \mathcal{F}, \mu, T)$ is a fixed weakly mixing system a.e. multiple recurrent and the systems $\left(Y_{j}, \mathcal{G}_{j}, m_{j}, S_{j}\right)$ are weakly mixing.

The method we will use relies on the following result, on the structure of pairwise independent joinings.

THEOREM 1. - Let $\left(X_{1}, \mathcal{B}_{1}, \mu_{1}, T_{1}\right)$ be an ergodic measure preserving system. Take $f \in L^{2}(\mu)$ and denote by $P f$ the projection of $f$ onto the vector space of those functions whose spectral measure is absolutely continuous with respect to Lebesgue measure on $\mathbb{T}$ and $P_{K_{1}} f$ the projection of fonto the Kronecker factor of $\left(X_{1}, \mathcal{B}_{1}, \mu_{1}, T_{1}\right)$. Let $w$ be a pairwise independent joining of $\left(X_{1}, \mathcal{B}_{1}, \mu_{1}, T_{1}\right)$ with two other ergodic dynamical systems $\left(X_{2}, \mathcal{B}_{2}, \mu_{2}, T_{2}\right)$ and $\left(X_{3}, \mathcal{B}_{3}, \mu_{3}, T_{3}\right)$. Then for all $f_{2}, f_{3} \in L^{\infty}$ we have

$$
\begin{aligned}
& \int f\left(x_{1}\right) f_{2}\left(x_{2}\right) f_{3}\left(x_{3}\right) \mathrm{d} w\left(x_{1}, x_{2}, x_{3}\right) \\
& =\int P_{K_{1}} f\left(x_{1}\right) f_{2}\left(x_{2}\right) f_{3}\left(x_{3}\right) \mathrm{d} w\left(x_{1}, x_{2}, x_{3}\right) \\
& \quad+\int P f\left(x_{1}\right) f_{2}\left(x_{2}\right) f_{3}\left(x_{3}\right) \mathrm{d} w\left(x_{1}, x_{2}, x_{3}\right) .
\end{aligned}
$$

This theorem is essentially a reformulation of Host's result [9]. The key observation incorporated in this reformulation is to notice that Host's proof can be applied to a single function with singular spectrum instead of assuming that the entire system has singular spectrum. The weakly 
mixing assumption on the sytems is also dropped. The focus on one single function is important for splitting a function into the sum of two functions one having a singular spectral measure the second an absolutely continuous spectral measure. As in [3] we can then deal with each function separately. This theorem is in our view of independent interest as not all pairwise independent joinings are independent.

We want to prove the following result.

THEOREM 2. - Let $(X, \mathcal{F}, \mu, T)$ be an a.e. multiple recurrent weakly mixing dynamical system, let $\left(A_{i}\right)_{1 \leqslant i \leqslant H}$ be measurable subsets of $X$ and let $\left(b_{1}, b_{2}, \ldots, b_{H}\right) \in \mathbb{Z}^{H}$ where the $b_{i}$ are all distinct and not equal to zero. Then for $\mu$ a.e. $x$ the sequence $a_{n}(x)=\left(\prod_{i=1}^{H} \mathbf{1}_{A_{i}}\left(T^{b_{i} n} x\right)\right)_{n}$ is a good universal weight for the pointwise convergence. Furthermore for such an $x$, for all ergodic dynamical systems $(Z, \mathcal{K}, \nu, R)$ for all $g \in L^{\infty}$ the averages

$$
\frac{1}{N} \sum_{n=1}^{N} a_{n}(x) g\left(R^{n} z\right)
$$

converge v a.e. to $\left(\prod_{i=1}^{H} \int \mathbf{1}_{A_{i}} \mathrm{~d} \mu\right) \int g \mathrm{~d} \nu$.

Proof. - Without the loss of generality we can and will assume that all dynamical systems are "standard" or regular in the following sense; that each system is of the form $(W, \mathcal{B}(W), \rho, P)$, where $W$ is compact metrizable, $\mathcal{B}(W)$ is the set of borelian subsets of $W, \rho$ is a probability measure on $\mathcal{B}(W)$ and $P$ a homeomorphism on $W$. This setting allows us to use in a crucial way continuous functions. Thus the system $(X, \mathcal{F}, \mu, T)$ is assumed to be "standard". We can observe that it is enough to prove that given $H$ a positive integer, $f_{1}, f_{2}, \ldots, f_{H}$ continuous functions, the sequence $a_{n}(x)=\left(\prod_{i=1}^{H} f_{i}\left(T^{b_{i} n} x\right)\right)_{n}$ is a good universal weight for the pointwise convergence. The general case for product of characteristic functions follows easily from it by approximation and the use of the maximal ergodic lemma.

The proof is given by induction on $H$.

\section{The case $H=1$}

It follows readily from [4] or [6]. The a.e. convergence $(v)$ of the averages

$$
\frac{1}{N} \sum_{n=1}^{N} f\left(T^{b n} x\right) g\left(R^{n} z\right)
$$


is clear for the systems $\left(X, \mathcal{F}, \mu, T^{b}\right), b \in \mathbb{Z}$. Furthermore if $T$ is weakly mixing and $\mathrm{b}$ is not equal to zero then the limit of these averages is equal to $\int f \mathrm{~d} \mu \int g \mathrm{~d} \nu$.

\section{The case $H$ positive integer}

Let us assume that the theorem holds for $H-1$. We are left proving that it holds then for $H$. We can remark that our induction assumption implies that the sequence

$$
\left(\prod_{i=2}^{H} \tilde{f}_{i}\left(T^{c_{i} n} x\right)\right)_{n}
$$

is a.e. a good universal weight for the pointwise convergence for all $\left(c_{2}, c_{3}, \ldots, c_{H}\right) \in \mathbb{Z}^{H-1}$ and all functions $\tilde{f}_{2}, \tilde{f}_{3}, \ldots, \tilde{f}_{H} \in L^{\infty}(\mu)$ (the $c_{i}$ are all distinct and none of them is equal to zero). Furthermore the limit of the averages

$$
\frac{1}{N} \sum_{n=1}^{N}\left(\prod_{i=2}^{H} \tilde{f}_{i}\left(T^{c_{i} n} x\right)\right) g\left(R^{n} z\right)
$$

is $v$ a.e. (z) equal to $\prod_{i=2}^{H} \int \tilde{f}_{i} \mathrm{~d} \mu \int g \mathrm{~d} \nu$.

We fix now $H$ continuous functions $f_{1}, f_{2}, \ldots, f_{H}$ and $\left(b_{1}, b_{2}\right.$, $\left.\ldots, b_{H}\right) \in \mathbb{Z}^{H}$ ( $b_{i}$ distinct and not equal to zero). By using a countable dense set of continuous functions in $\mathcal{C}\left(X^{H}\right)$, set of continuous functions on $X^{H}$, and the a.e. multiple recurrence property of the system $(X, \mathcal{F}, \mu, T)$, we can find a set of full measure $\widetilde{X}$ such that for $x \in \widetilde{X}$ we have

(4) $\lim _{N} \frac{1}{N} \sum_{n=1}^{N} F\left(T^{b_{1} n} x, T^{b_{2} n}, \ldots, T^{b_{H} n} x\right)=\int F\left(x_{1}, x_{2}, \ldots, x_{H}\right) \mathrm{d} \mu^{H}$

for all $F \in \mathcal{C}(X)$ where $\mu^{H}$ is the $H$-product measure $\mu \otimes \mu \otimes \cdots \otimes \mu$ on $\mathcal{B}\left(X^{H}\right)$. In particular we have for $x \in \widetilde{X}$

$$
\lim _{N} \frac{1}{N} \sum_{n=1}^{N}\left(\prod_{i=1}^{H} f_{i}\left(T^{b_{i} n} x\right)\right)=\prod_{i=1}^{H} \int f_{i} \mathrm{~d} \mu .
$$

By using our induction assumption (3) and the previous argument for (4) and (5) we can obtain the following; 
(6) there exists a subset of full measure $\mathcal{X}_{1} \subset \widetilde{X}$ such that if $x \in \mathcal{X}_{1}$ for all ergodic dynamical systems $(Z, K, \nu, R)$ for all $G \in \mathcal{C}(X \times Z)$

$$
\lim _{N} \frac{1}{N} \sum_{n=1}^{N} G\left(T^{b_{1} n} x, R^{n} z\right)=\int G\left(x_{1}, z\right) \mathrm{d} \mu \otimes v
$$

for all $z$ in a subset of $Z, Z_{1}$ of full measure,

(7) there exists a subset of full measure $\mathcal{X}_{2} \subset \tilde{X}$ such that if $x \in$ $\mathcal{X}_{2}$ for all "standard" or "regular" ergodic dynamical systems $(Z, \mathcal{K}, v, R)$ for all $Q \in \mathcal{C}\left(X^{H-1} \times Z\right)$ we have

$$
\begin{aligned}
\lim _{N} & \frac{1}{N} \sum_{n=1}^{N} Q\left(T^{b_{2} n} x, T^{b_{3} n} x, \ldots, T^{b_{H} n} x, R^{n} z\right) \\
\quad & \int Q\left(x_{2}, x_{3}, \ldots, x_{H}, z\right) \mathrm{d} \mu^{H-1} \otimes v
\end{aligned}
$$

for all $z$ in a subset of $Z, Z_{2}$ of full measure.

We want to show that $\mathcal{X}=\mathcal{X}_{1} \cap \mathcal{X}_{2}$ is the set of full measure for which the sequence $a_{n}(x)=\left(\prod_{i=1}^{H} f_{i}\left(T^{b_{i} n} x\right)\right)$ is good universal. To prove this we now consider an ergodic dynamical system $(Z, \mathcal{K}, v, R)$ that we also assume to be "standard" or "regular". It will be enough to show that for $x \in \mathcal{X}$ the averages

(8) $\frac{1}{N} \sum_{n=1}^{N}\left(\prod_{i=1}^{H} f_{i}\left(T^{b_{i} n} x\right)\right) g\left(R^{n} z\right)$ converge a.e. (v) for all $g \in \mathcal{C}(Z)$.

Again the general conclusion will follow by density and approximation. By the spectral decomposition we can write $g$ as the following sum: $g=\int g \mathrm{~d} v+g^{S}+P g$ where $g^{S}$ and $P g$ are $L^{2}$ functions with their respective spectral measures $\sigma_{g} s, \sigma_{P g}$ having the following properties

(a) $\sigma_{g} s$ is singular with respect to Lebesgue measure $m$ on $\mathbb{T}$;

(b) $\sigma_{P g}$ is absolutely continuous with respect to Lebesgue measure $m$ on $\mathbb{T}$.

Hence to establish (8) we just need to prove that the limits

$$
\begin{aligned}
& \lim \frac{1}{N} \sum_{n=1}^{N}\left(\prod_{i=1}^{H} f_{i}\left(T^{b_{i} n} x\right)\right) \cdot \int g \mathrm{~d} \nu, \\
& \lim \frac{1}{N} \sum_{n=1}^{N}\left(\prod_{i=1}^{H} f_{i}\left(T^{b_{i} n} x\right)\right) g^{S}\left(R^{n} z\right)
\end{aligned}
$$


and

$$
\lim _{N} \frac{1}{N} \sum_{n=1}^{N}\left(\prod_{i=1}^{H} f_{i}\left(T^{b_{i} n} x\right)\right)(P g)\left(R^{n} z\right)
$$

exist a.e. (v).

The limit in (9) exists because of (4). To establish (10) we need to observe first that given $g^{S} \in L^{2}(v)$ we can find a sequence of continuous function $\left(g_{j}\right)_{j}\left(g_{j} \in \mathcal{C}(Z)\right)$ such that $\sum_{j=1}^{\infty}\left\|g^{S}-g_{j}\right\|_{1}<\infty$. This is clear by density and approximation. By the maximal inequality we conclude that for all $\lambda>0$

$$
\sum_{j=1}^{\infty} v\left\{z: \sup _{n}\left(\frac{1}{n} \sum_{k=1}^{n}\left|\left(g^{S}-g_{j}\right)\left(R^{n} z\right)\right|\right)>\lambda\right\} \leqslant \frac{1}{\lambda} \sum_{j=1}^{\infty}\left\|g^{S}-g_{j}\right\|_{1} .
$$

By using the Borel-Cantelli lemma we derive

$$
\limsup _{j} \frac{1}{n} \sum_{k=1}^{n}\left|\left(g^{S}-g_{j}\right)\left(R^{n} z\right)\right|=0 \quad \text { a.e. }(z) \text {. }
$$

This equation in turn implies that for any bounded sequence $a_{n}(x)$

$$
\limsup _{j} \frac{1}{n} \sum_{k=1}^{n}\left|a_{n}(x)\right|\left|\left(g^{S}-g_{j}\right)\left(R^{n} z\right)\right|=0 \quad \text { a.e. }(z) .
$$

(The set $Z_{3}$ on which (13) holds is independent of the sequence $a_{n}(x)$.)

We want to prove (10) by showing that

$$
\begin{aligned}
& \varlimsup \frac{1}{N} \sum_{n=1}^{N}\left(\prod_{i=1}^{H} f_{i}\left(T^{b_{i} n} x\right)\right) g^{S}\left(R^{n} z\right) \\
& =\underline{\lim _{1}} \frac{1}{N} \sum_{n=1}^{N}\left(\prod_{i=1}^{H} f_{i}\left(T^{b_{i} n} x\right)\right) g^{S}\left(R^{n} z\right) \\
& =0 \quad \text { (for } z \in Z_{1} \cap Z_{2} \cap Z_{3} \text { ). }
\end{aligned}
$$

We consider the lim sup first.

By using the a.e. $(v)$ boundedness of the sequence

$$
\frac{1}{N} \sum_{n=1}^{N}\left(\prod_{i=1}^{H} f_{i}\left(T^{b_{i} n} x\right)\right) g^{S}\left(R^{n} z\right)
$$

we can find a subsequence $\left(N_{k}^{x}(z)\right)_{k}$ such that 


$$
\lim _{k} \frac{1}{N_{k}^{x}(z)} \sum_{n=1}^{N_{k}^{x}(z)}\left(\prod_{i=1}^{H} f_{i}\left(T^{b_{i} n} x\right)\right) g^{S}\left(R^{n} z\right)=\varlimsup \frac{1}{N} \sum_{n=1}^{N} a_{n}(x) g^{S}\left(R^{n} z\right)
$$

(where we denote by $a_{n}(x)$ the quantity $\prod_{i=1}^{H} f_{i}\left(T^{b_{i} n} x\right)$ ).

From the sequence $\left(N_{k}^{x}(z)\right)$ we can extract by a diagonal process a subsequence $\left(M_{k}^{x}(z)\right)$ such that

$$
\lim _{k} \frac{1}{M_{k}^{x}(z)} \sum_{n=1}^{M_{k}^{x}(z)} V\left(T^{b_{1} n} x, T^{b_{2} n} x, \ldots, T^{b_{H} n} x, R^{n} z\right)
$$

exists for all continuous functions $V \in \mathcal{C}\left(X^{H} \times Z\right)$. This limit (14) guarantees by the Riesz representation theorem the existence of a probability measure $\omega^{x, z}$ such that

$$
\begin{aligned}
& \lim _{k} \frac{1}{M_{k}^{x}(z)} \sum_{n=1}^{M_{k}^{x}(z)} H\left(T^{b_{1} n} x, T^{b_{2} n} x, \ldots, T^{b_{H} n} x, R^{n} z\right) \\
& \quad=\int H\left(x_{1}, x_{2}, \ldots, x_{H}, t\right) \mathrm{d} \omega^{x, z} .
\end{aligned}
$$

We claim that this measure $\omega^{x, z}$ is a pairwise independent joining of three systems $(\alpha)\left(X, \mathcal{B}(X), \mu, T^{b_{1}}\right),(\beta)\left(X^{H-1}, \mathcal{B}\left(X^{H-1}\right), \mu^{H-1}, T^{b_{2}} \times T^{b_{3}} \times\right.$ $\left.\cdots \times T^{b_{H}}\right)$, and $(\gamma)(Z, K, v, R)$. Let us see why; (6) and (7) based on our induction assumption imply that the projections $\operatorname{proj}_{(\alpha),(\gamma)} \omega^{x, z}$ and $\operatorname{proj}_{(\beta),(\gamma)} \omega^{x, z}$ are respectively $\mu \otimes \nu$ and $\mu^{H-1} \otimes \nu$. Finally, (4) guarantees the third projection $\operatorname{proj}_{(\alpha),(\beta)} \omega^{x, z}$ to be $\mu^{H}$. Theorem 1 tells us that

$$
\int\left(\prod_{i=1}^{H} f_{i}\left(x_{i}\right)\right) g^{S}(t) \mathrm{d} w^{x, z}=\left(\prod_{i=1}^{H} \int f_{i} \mathrm{~d} \mu\right)\left(\int g^{s} \mathrm{~d} v\right)+0
$$

(as $P\left(g^{S}\right)=0$ and $\left.P_{K_{i}}\left(f_{i}\right)=\int f_{i} \mathrm{~d} \mu\right)$. But $\int g^{S} \mathrm{~d} \nu=0$, hence we have

$$
\int \prod_{i=1}^{H} f_{i}\left(x_{i}\right) \cdot g^{S}(t) \mathrm{d} w^{x, z}=0 .
$$

It remains to show that the integral in (15) is in fact the limsup we started with. This can be achieved by (13). We know by the construction of $w^{x, z}$ that for all $g_{j}$ continuous function in $\mathcal{C}(Z)$ we have

$$
\lim _{k} \frac{1}{M_{k}^{x}(z)} \sum_{n=1}^{M_{k}^{x}(z)}\left(\prod_{i=1}^{H} f_{i}\left(T^{b_{i} n} x\right)\right) g_{j}\left(R^{n} z\right)=\int\left(\prod_{i=1}^{H} f_{i}\left(x_{i}\right)\right) g_{j}(t) \mathrm{d} w^{x, z} .
$$


By (13) and for $z$ in $Z_{1} \cap Z_{2} \cap Z_{3}$ we have for a sequence $\left(g_{j}\right)$ of continuous functions such that

$$
\begin{aligned}
& \sum_{j=1}^{\infty}\left\|g^{S}-g_{j}\right\|_{1}<\infty, \\
& \limsup _{j}\left|\frac{1}{n} \sum_{k=1}^{n}\right| a_{n}(x)||\left(g^{S}-g_{j}\right)\left(R^{n} z\right)||=0 .
\end{aligned}
$$

Thus

$$
\lim _{j} \int\left(\prod_{i=1}^{H} f_{i}\left(x_{i}\right)\right) g_{j}(t) \mathrm{d} w^{x, z}=\int\left(\prod_{i=1}^{H} f_{i}\left(x_{i}\right)\right) g^{S}(t) \mathrm{d} w^{x, z}
$$

because the projection $\operatorname{proj}_{(\gamma)} w^{x, z}=v$ and

$$
\begin{aligned}
\lim _{k} & \frac{1}{M_{k}^{x}(z)} \sum_{n=1}^{M_{k}^{x}(z)}\left(\prod_{i=1}^{H} f_{i}\left(T^{b_{i} n} x\right)\right) g^{S}\left(R^{n} z\right) \\
= & \lim _{j} \int\left(\prod_{i=1}^{H} f_{i}\left(x_{i}\right)\right) g_{j}(t) \mathrm{d} w^{x, z} \\
= & \int\left(\prod_{i=1}^{H} f_{i}\left(x_{i}\right)\right) g^{S}(t) \mathrm{d} w^{x, z} .
\end{aligned}
$$

Hence for $x \in \mathcal{X}$ and $z \in Z_{3} \cap Z_{1} \cap Z_{1}$ we have

$$
\varlimsup \frac{1}{N} \sum_{n=1}^{N}\left(\prod_{i=1}^{H} f_{i}\left(T^{b_{i} n} x\right)\right) g^{S}\left(R^{n} z\right)=0 .
$$

We can see that a similar argument will give us

$$
\varliminf \frac{1}{N} \sum_{n=1}^{N}\left(\prod_{i=1}^{H} f_{i}\left(T^{b_{i} n} x\right)\right) g^{S}\left(R^{n} z\right)=0 .
$$

Thus (10) is proved.

To finish the proof of Theorem 2 we need to show (11). We look at the closed subspace of $L^{2}(v), E$, of functions $h \in L^{2}(v)$ such that $\sigma_{h} \ll m$. The function $P g$ belongs to $E$. To prove (11) it is enough to prove that it holds for a dense subset of $E$. This subset is provided by the functions $h$ such that $\mathrm{d} \sigma_{h}=\rho \mathrm{d} m$ where $m$ is Lebesgue measure and $\rho$ is a uniformly bounded function on $\mathbb{T}$, i.e., $\|\rho\|_{\infty} \leqslant C<\infty$. For each positive integer $N$ we have 


$$
\begin{aligned}
\int\left|\frac{1}{N} \sum_{n=1}^{N} a_{n}(x) h\left(R^{n} z\right)\right|^{2} \mathrm{~d} \nu & =\frac{1}{N^{2}} \int\left|\sum_{n=1}^{N} a_{n}(x) \mathrm{e}^{2 \pi \mathrm{i} n \varepsilon}\right|^{2} \rho(\varepsilon) \mathrm{d} m \\
& \leqslant C \frac{1}{N^{2}} \sum_{n=1}^{N}\left|a_{n}(x)\right|^{2} .
\end{aligned}
$$

Hence the sequence $\frac{1}{N^{2}} \sum_{n=1}^{N} a_{n}(x) \cdot h\left(R^{n} z\right)$ converges a.e. (v) to 0 . Finally by a classical argument used in [7] we conclude that the sequence $\frac{1}{N} \sum_{n=1}^{N} a_{n}(x) h\left(R^{n} z\right)$ converges a.e. $(v)$ to 0 .

In this section we want to show that we can extend Theorem 2 in another direction. Our goal is to show that weights of the form

$$
\left(\prod_{i=1}^{H} f_{i}\left(T^{b_{i} n} x\right)\right) \cdot\left(\prod_{j=1}^{J} g_{j}\left(S_{j}^{n} y_{j}\right)\right),
$$

where the systems $\left(Y_{j}, \mathcal{G}_{j}, S_{j}, v_{j}\right)$ are generated in the universal way given in the introduction, are also good universal for the pointwise convergence

THEOREM 3. - Let $(X, \mathcal{F}, \mu, T)$ be an a.e. multiple recurrent weakly mixing dynamical system, $H$ a positive integer and let $f_{1}, f_{2}, \ldots, f_{H}$ be $H L^{\infty}$ functions. Consider also $\left(b_{1}, b_{2}, \ldots, b_{H}\right) \in \mathbb{Z}^{H}$ where the $b_{i}$ are all distinct and none is equal to zero. There exists a set $\mathcal{X}$ of full measure such that if $x \in \mathcal{X}$ then we have the following:

1) For all weakly mixing systems $\left(Y_{1}, \mathcal{G}_{1}, S_{1}, v_{1}\right)$ and $g_{1} \in L^{\infty}\left(v_{1}\right)$ there exists a set $\widetilde{Y}_{1}$ of full measure such that for $y_{1} \in \widetilde{Y}_{1}$ we have the following:

2) For all weakly mixing systems $\left(Y_{2}, \mathcal{G}_{2}, S_{2}, \nu_{2}\right)$ and $g_{2} \in L^{\infty}\left(\nu_{2}\right)$ there exists a set $\widetilde{Y}_{2}$ of full measure such that for $y_{2} \in \widetilde{Y}_{2}$ we have the following:

J) For all weakly mixing systems $\left(Y_{J}, \mathcal{G}_{J}, S_{J}, \nu_{J}\right)$ and all $g_{J} \in L^{\infty}\left(\nu_{J}\right)$ the following holds: 
(1) The sequence

$$
a_{n}\left(x, y_{1}, y_{2}, \ldots, y_{J}\right)=\left(\prod_{i=1}^{H} f_{i}\left(T^{b_{i} n} x\right)\right) \cdot\left(\prod_{j=1}^{J} g_{j}\left(S_{j}^{n} y_{j}\right)\right)
$$

is $v_{J}$ (a.e. $y_{J}$ ) a good universal weight for the pointwise convergence.

Proof. - For the sake of simplicity we will treat only the case $J=2$. The reader can check that the arguments extend without difficulty to the general case of $J$ positive integer. As in the proof of Theorem 2 we can and will assume that all systems are "standard" or "regular". As the ideas are mainly the same as in the proof of Theorem 4 we only sketch the arguments.

Step 1: We use Theorem 1 to guarantee the existence of a set of full measure $X_{1}$ such that if $x \in X_{1}$ the sequence $\left(\prod_{i=1}^{H} f_{i}\left(T^{b_{i} n} x\right)\right)$ is $\mu$ a.e. good universal.

Step 2: We pick a weakly mixing system $\left(Y_{1}, \mathcal{G}_{1}, S_{1}, v_{1}\right)$ and $g_{1} \in$ $\mathcal{C}\left(Y_{1}\right)$. By the result in [4] or [6] we pick a set of full measure $\widetilde{Y}_{1}$ such that for all $y_{1} \in \widetilde{Y}_{1}$ the sequence $\left(g_{1}\left(S_{1}^{n} y_{1}\right)\right)_{n}$ is a good universal weight.

Step 3: We find subsets $X_{2}$ of $X_{1}$ and $\bar{Y}_{1}$ of $\widetilde{Y}_{1}$ such that the sequence

$$
\left(\left(\prod_{i=1}^{H} f_{i}\left(T^{b_{i} n} x\right)\right) g_{1}\left(S_{1}^{n} y_{1}\right)\right)_{n}
$$

is good universal for the pointwise convergence. At this stage we pick any weakly mixing system $\left(Y_{2}, \mathcal{G}_{2}, v_{2}, S_{2}\right)$ and $g_{2} \in \mathcal{C}\left(Y_{2}\right)$. We use the spectral decomposition to write $g_{2}$ as $\int g_{2} \mathrm{~d} \nu_{2}+g_{2}^{S}+P g_{2}$ where $\sigma_{g_{2}}^{S} \perp m$ and $\sigma_{P_{2}} \ll m$.

The averages for $P g_{2}$ are handled in the same way (using the series and a dense subset $E_{2}$ of $L^{2}\left(v_{2}\right)$ ). For $g_{2}^{S}$ we can consider the $\overline{l i m}$ and $\underline{\mathrm{lim}}$. We construct a joining which we make pairwise independent at the expense of restricting the set $\bar{Y}_{1}$ and $X_{2}$ but maintaining the total mass to be equal to 1 .

Theorem 2 guarantees the existence of the limit of these averages along an appropriate subsequence to be equal to zero. Similar arguments for the

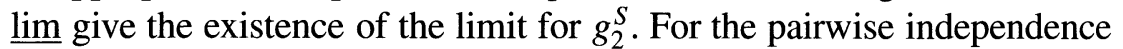
the three systems considered are $\left(X^{H}, \mathcal{B}\left(X^{H}\right), T^{b_{1}} \times T^{b_{2}} \times \cdots \times T^{b_{H}}\right)$, $\left(Y_{1}, \mathcal{B}\left(Y_{1}\right), v_{1}, S_{1}\right)$ and $\left(Y_{2}, \mathcal{B}\left(Y_{2}\right), v_{2}, S_{2}\right)$. The universality property is also ensured by Step 1 and Step 2 . 
Step 4: We proceed similarly as in Step 3, this time to prove that for appropriate universal subsets of $X_{2}, \bar{Y}_{1}$ and $Y_{2}$ the sequence $\left(\prod_{i=1}^{H} f_{i}\left(T^{b_{i} n} x\right)\right)\left(g_{1}\left(S_{1}^{n} y_{1}\right) g_{2}\left(S_{2}^{n} y_{2}\right)\right)$ is good universal for the pointwise convergence. The arguments are the same as previously. We use Step 3, the spectral decomposition, Theorem 4 and the result in [4] or [6] to prove as previously the universality property expected.

Remark. - The results in this paper were obtained some years ago. Since then one extension of Theorem 2 has appeared in [11] for the case $H=0, J$ any positive integer and $S_{j}$ ergodic.

\section{REFERENCES}

[1] Assani I., A double return times ergodic theorem, Preprint, 1997.

[2] Assani I., Strong laws for weighted sums of iid random variables, Duke Math. J. 88 (1997) 217-246.

[3] Assani I., Multiple recurrence and almost sure convergence for weakly mixing dynamical systems, Israel J. Math. 103 (1998) 111-124.

[4] Bourgain J., Return times sequences of dynamical systems, Preprint, IHES, March 1988.

[5] Bourgain J., Double recurrence and almost sure convergence, J. für die Reine und Angewandte Math. 404 (1990) 140-161.

[6] Bourgain J., Furstenberg H., Katznelson Y., Ornstein D., Appendix to J. Bourgain, Pointwise Ergodic Theorems for Arithmetic Sets, IHES, 1989.

[7] Bellow A., Losert V., The weighted pointwise ergodic theorem and the individual ergodic theorem along subsequences, Trans. Amer. Math. Soc. 288, 307-345.

[8] Furstenberg H., Recurrence in Ergodic Theory and Combinatorial Number Theory, Princeton University Press, Princeton, NJ, 1981.

[9] Host B., Mixing of all orders and pairwise independent joinings of systems with singular spectrum, Israel J. Math. 76 (1991) 289-298.

[10] Lesigne E., Sur la convergence ponctuelle de certaines moyennes ergodiques, Comptes Rendus de l'Académie des Sciences 298 (1984) 425-428.

[11] Rudolph D., Fully generic sequences and a multiple term return times theorem, Invent. Math. 131 (1998) 199-228. 